\title{
Discrete scale invariance effects in the nonequilibrium critical behavior of the Ising magnet on a fractal substrate
}

\author{
M. A. Bab, ${ }^{1,2, *}$ G. Fabricius, ${ }^{1,3, \dagger}$ and E. V. Albano ${ }^{1, \sharp}$ \\ ${ }^{1}$ Instituto de Investigaciones Fisicoquímicas Teóricas y Aplicadas, Facultad de Ciencias Exactas, \\ Universidad Nacional de La Plata, Sucursal 4, CC 16 (1900) La Plata, Argentina \\ ${ }^{2}$ Departamento de Física, Facultad de Ciencias Exactas, Universidad Nacional de La Plata, La Plata, Argentina \\ ${ }^{3}$ Abdus Salam International Centre for Theoretical Physics, Trieste, Italy
}

(Received 9 March 2006; published 26 October 2006)

\begin{abstract}
The nonequilibrium critical dynamics of the Ising magnet on a fractal substrate, namely the Sierpinski carpet with Hausdorff dimension $d_{H}=1.7925$, has been studied within the short-time regime by means of Monte Carlo simulations. The evolution of the physical observables was followed at criticality, after both annealing ordered spin configurations (ground state) and quenching disordered initial configurations (high temperature state), for three segmentation steps of the fractal. We have obtained evidence showing that during these relaxation processes both the growth and the fragmentation of magnetic domains become influenced by the hierarchical structure of the substrate. In fact, the interplay between the dynamic behavior of the magnet and the underlying fractal leads to the emergence of a logarithmic-periodic oscillation, superimposed to a power law, which has been observed in the time dependence of both the decay of the magnetization and its logarithmic derivative. These oscillations have been carefully characterized in order to determine the critical temperature of the second-order phase transition and the critical exponents corresponding to the short-time regime. The effects of the substrate can also be observed from the dependence of the effective critical exponents on the segmentation step. The exponent $\theta$ of the initial increase of the magnetization has also been obtained and the results suggest that it would be almost independent of the fractal dimension of the susbstrate, provided that $d_{H}$ is close enough to $d=2$. The oscillations have been discussed within the framework of the discrete scale invariance of the substrate.
\end{abstract}

DOI: 10.1103/PhysRevE.74.041123

PACS number(s): 64.60.Ht, 05.45.Df, 75.10.Hk, 02.70.Uu

\section{INTRODUCTION}

The study and characterization of continuous phase transitions occurring on fractal substrates have attracted much attention during the past decades in the research fields of statistical physics and materials science. The very wellknown general belief in universality states that for a given symmetry of the order parameter and range of interactions, the critical behavior only depends on the dimensionality $d$, i.e., the influence of the underlying structure becomes negligible at the critical point when the correlation length is much larger than the cell spacing. In particular, Ising models embedded in different lattices in the absence of external magnetic fields, with short-range interactions and for a given $d$ $>1$, exhibit continuous phase transitions characterized by identical critical exponents, with the critical temperature decreasing to zero at the lower critical dimension $d=1$.

Instead of the replication of an elementary cell by translation, fractals are constructed by the iteration of a generating cell, consequently the topological details of the generating cell are present at any scale. In this way, the critical exponents also depend on other geometric and topological parameters, such as the ramification and lacunarity of the fractal, and the universality is said to be weak [1,2]. It has been shown that a second-order phase transition at nonzero tem-

\footnotetext{
*Electronic address: mbab@inifta.unlp.edu.ar

†Electronic address: fabriciu@ fisica.unlp.edu.ar

‡Electronic address: ealbano@inifta.unlp.edu.ar
}

perature occurs only if the fractal substrate has an infinite ramification order [3-5]. The lacunarity was introduced by Mandelbrot such as the measure of the deviation from the translational invariance of the fractal [6], and subsequently different proposals have been formulated in order to quantify this concept. Some of them imply that the lacunarity cannot be reduced to a number and suggest analytical expressions based on the fluctuations of the mass scaling $m(R) \propto R^{d_{H}}$, where $m(R)$ is the mass inside a sphere of radius $R$ centered in the fractal and $d_{H}$ is the fractal Hausdorff dimension [7-9]. Throughout the present paper we refer to lacunarity qualitatively as a property related to the size and distribution of holes in the fractal.

Direct quantitative studies of the Ising model on fractal substrates have shown that the values of the ratio of critical exponents $\gamma / \nu$ depend upon the lacunarity and indicate that by increasing the lacunarity one has to introduce higher corrections in the finite-size scaling treatment associated with the critical behavior of physical observables [2].

Most of the previous work on this topic was based on the same class of fractals, built in the same way as the standard Sierpinski carpet $[\mathrm{SC}(b, c)]$, which have an infinite ramification order. A hypercube in $d$ dimensions is segmented into $b^{d}$ subhypercubes and $c^{d}$ of them are then removed, this segmentation process is iterated on the remaining subhypercubes. The mathematical fractal is obtained after an infinite number of segmentation steps $(k)$. However, for practical purposes, a fractal is constructed by applying a finite number of segmentation steps. So, the physical observables show not only finite-size effects caused by the size of the system, but 


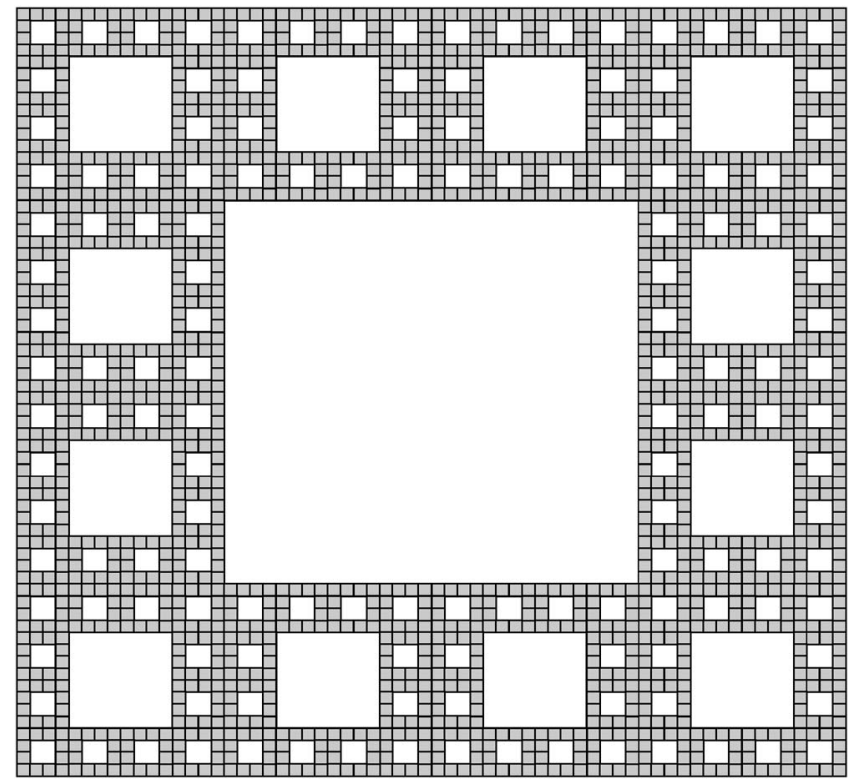

FIG. 1. Sketch of the Sierpinski carpet SC $(4,2)$ iterated up to the $k=3$ segmentation step. Spins are placed at the center of filled squares.

also a lower cutoff due to the segmentation step. As a matter of fact, results obtained by performing Monte Carlo simulations depend on the number of segmentation steps, so it is interesting to know the way these observables converge to the ones expected for the mathematical fractal. The key point here is that in order to obtain critical exponents from simulations in equilibrium, one has to apply finite-size scaling analysis that is hindered by topological scaling corrections. These corrections and the critical slowing down increase when the fractal dimension decreases towards one. Furthermore, due to these effects the segmentation steps studied could not be large enough to truly observe the asymptotic behavior. So, under these circumstances it is not surprising that also the critical exponents could not be easily obtained $[2,10]$. Furthermore, Pruessner et al. [12] have questioned the validity of finite-size scaling on fractal substrates arguing that each segmentation step represents a new thermodynamic system that cannot be treated as a scaled version of the previous one.

During the last years, nonequilibrium critical dynamics has been developed and a dynamic scaling form was found that is already valid in the short-time regime $[13,14]$. In addition to the dynamic exponent $z$, the static exponents, originally defined in equilibrium, also appear in the short-time scaling form. This fact leads to new methods for the numerical measurement of all the static and dynamic critical exponents, as well as the critical temperature. Since the measurements now are carried out within the short-time regime, they do not suffer from critical slowing down. Because of the small nonequilibrium correlation length, it is also easy to overcome finite-size effects. Due to the above-mentioned advantages the nonequilibrium critical dynamics within the short-time regime is a promising tool for the study of continuous phase transitions on fractal substrates. Hitherto, the topological effects on the short-time dynamic behavior and the possible corrections to scaling have not been systematically investigated. Recently, we have reported soft oscillatory deviations from the power-law decay of the magnetization in the short-time regime [15] for the Ising model on the $\operatorname{SC}(3,1)$ with $d_{H}=1.8927$. On the other hand, logarithmicperiodic oscillatory deviations in the behavior of physical observables have been reported for several systems that present fractal characteristics embedded into the dynamics of the model and/or the substrate. Some examples are the wave propagation in fractal systems [16], the Blume-Capel model on the Sierpinski gasket [17], the dynamics of biological systems such as the bronchial tree [18], proteins [19], magnetic and resistive effects on a system of wires connected along the Sierpinski gasket [16], and random walks through fractal environments [20].

The main purpose of the present paper is to work out the connection between a discrete scaling symmetry invariance-as it is present in hierarchically built fractal structures-and the corresponding symmetry in the time domain for the relaxation behavior of an Ising model defined on such a substrate. For this purpose we have performed extensive numerical Monte Carlo simulations of the nonequilibrium short-time critical dynamics of the Ising model on the $\mathrm{SC}(4,2)$. Three different segmentation steps are investigated in order to show how the lower cutoff due to the finitesegmentation step affects both the effective critical exponents and effective critical temperature. This procedure also allows us to study the convergence of the measured values toward those expected for the mathematical fractal. We have observed that the scaling law that describes the approach to equilibrium of the magnetization as a function of time during the annealing from a completely ordered state exhibits welldefined logarithmic-periodic oscillations. We argue that the period of these oscillations is related to the scale ratio of successive segmentation steps via the dynamic exponent $z$.

In Sec. II we introduce the model, we give the simulation details and remarks on the main features of the short-time dynamic scaling. In Sec. III we present, analyze, and discuss the short-time dynamic behavior of systems starting from both disordered (Sec. III A) and ordered initial states (Sec. III B). Critical exponents obtained under these two conditions are compared. In Sec. III C we discuss the characteristics of the substrate and their influence on the dynamic critical behavior of the physical observables. In Sec. IV we present our conclusions.

\section{THE MODEL AND THE METHODS}

\section{A. The Ising model on Sierpinski carpets}

Figure 1 shows a sketch of the fractal substrate used in the present work, namely the $\mathrm{SC}(4,2)$, for the segmentation step $k=3$. The Hausdorff dimension of the mathematical fractal is given by $d_{H}=\frac{\log \left(4^{2}-2^{2}\right)}{\log 4} \simeq 1.7925$. In the case of the fractal, the convergence of relevant properties towards the thermodynamic limit occurs when the structure is constructed by the iterative process. Such convergence is reflected in the dependence of the mean number of nearest neighbors per site on the segmentation step, which converges to a constant value 
as $k$ goes to infinity. In order to study the critical behavior by means of Monte Carlo simulations it is necessary to simulate large enough segmentation steps to ensure that the asymptotic region is reached. For the $\mathrm{SC}(4,2)$ the deviation of the mean number of next-nearest neighbors from the value corresponding to the thermodynamic limit (mathematical fractal), using periodic boundary conditions and determined by means of the transfer-matrix method, becomes negligible for $k \geqslant 5[10,11]$.

As mentioned, the studied fractal has an infinite ramification order, which implies that the Ising model would exhibit a continuous phase transition at finite temperature. In order to perform the simulation the spins were placed at the center of the occupied subsquares and consequently, the number of spins increases as a power law of the lattice size with an exponent given by $d_{H}$. The Hamiltonian of the system is then given by

$$
H=-J \sum_{\langle i, j\rangle} s_{i} s_{j},
$$

where $s_{i}$ assumes the values \pm 1 , the sum runs over all interacting first-neighbor pairs, and the positive exchange coupling constant $J$ corresponds to the ferromagnetic case.

\section{B. Short-time dynamic behavior at second-order phase transitions}

In this section we give a brief summary on the short-time dynamic (STD) method for characterization of continuous phase transitions. According to STD arguments [13], a magnetic system at high temperature and with a small magnetization $m_{0} \ll 1$ suddenly quenched to the critical temperature $T_{c}$ presents a universal dynamical evolution, which sets right after a microscopic time scale $t_{\text {mic }}$, large enough in the microscopic sense but still very small in the macroscopic sense. If during this time interval the nonequilibrium correlation length remains smaller than the system size the short-time dynamics is free of finite-size effects. According to the shorttime dynamic scaling $[14,21,22]$ the magnetization, its second moment, and the autocorrelation function should follow power-law scaling relations given by

$$
\begin{gathered}
M(t)=\left\langle\frac{1}{N} \sum_{i=1}^{N} s_{i}\right\rangle \sim m_{0} t^{\theta} F\left(t^{\theta+\beta / v z} m_{0}\right), \\
M^{2}(t)=\left\langle\left(\frac{1}{N} \sum_{i=1}^{N} s_{i}\right)^{2}\right\rangle \propto t^{\left[d_{e f f} / z-2 \beta / v z\right]}, \\
A(t)=\left\langle\frac{1}{N} \sum_{i=1}^{N} s_{i}(t) s_{i}(0)\right\rangle \propto t^{-\lambda}, \quad \text { with } \lambda=\left(\frac{d_{e f f}}{z}-\theta\right),
\end{gathered}
$$

respectively. Here $\langle\cdots\rangle$ denotes the average over samples, $N$ is the total number of spins in the sample, $\theta$ is the exponent of the initial increase in the magnetization, and $d_{e f f}$ is the scaling dimension whose value may be different than $d_{H}$. Also, $\beta$ and $\nu$ are the standard-usually defined in equilibrium - order parameter and correlation length critical exponents, respectively. The scaling function behaves as $F(x) \sim 1$ for $x \rightarrow 0$ and $F(x) \sim \frac{1}{x}$ for $x \rightarrow \infty$. Extrapolating the results to $m_{0}=0$, the exponent $\theta$ of the initial increase in the magnetization, which is a new nontrivial critical exponent, can be obtained. We remark that $M^{2}(t)$ and $A(t)$ have to be measured starting from fully uncorrelated configurations with strictly zero magnetization, i.e., for $T=\infty$. One should also expect that the generalized hyperscaling relationship given by $d_{e f f}-\frac{2 \beta}{\nu}=\gamma$ would hold for the Ising model on a fractal substrate [see also Eq. (3)].

For a dynamic relaxation from a completely ordered state $\left(m_{0}=1\right)$ corresponding to the ground state at $T=0$, annealed to $T_{c}$, the magnetization, the logarithmic derivative of the magnetization with respect to the reduced temperature $\tau$ $=\frac{T-T_{c}}{T_{c}}$, and the second-order Binder cumulant should follow power laws in time, namely

$$
\begin{gathered}
M(t) \propto t^{-\beta / v z}, \\
V_{\tau}(t)=\left.\partial_{\tau}(\ln M(t, \tau))\right|_{\tau=0} \propto t^{1 / v z}, \\
U(t)=\frac{M^{2}(t)}{[M(t)]^{2}}-1 \propto t^{d_{e f f} / z},
\end{gathered}
$$

respectively. For $T \neq T_{c}$, but within the critical region, the power-law behavior is modified by a scaling function, which for the magnetization is given by $M^{*}\left(t^{1 / v z} \tau\right)$. So, this fact can be used to determine the critical temperature from the localization of the best power-law behavior, i.e., the smallest deviations from a power law.

We have found that the dynamic evolution of the magnetization when the system is annealed from $T=0$ to a higher temperature $T$ close to the critical point is more sensitive to this final temperature than when the system is quenched from $T=\infty$. Furthermore, due to the large initial value of $M$ and its slow decrease upon annealing, the statistical fluctuations are less prominent, and therefore it would be expected that the effects due to the underlying substrate may be easier to detect.

\section{Monte Carlo simulations}

In order to study the effects of the fractal substrate on the STD and the convergent behavior of the critical exponents with the segmentation step, we carried out Monte Carlo simulations for segmentation steps $k=4,5$, and 6 (system size $L=256,1024$, and 4096, respectively) using periodic boundary conditions. Simulations started either from the complete ordered state or from a disordered state with zero or a small magnetization. In the last case the initial magnetization $m_{0}$ was settled by flipping, in a random disordered configuration, a definite number of spins placed at randomly chosen sites in order to get the desired value of $m_{0}$. The time evolution of the system was updated by means of a Metropolis algorithm using the Marsaglia-Zanan pseudorandom number generator [23]. The time unit, defined as a Monte Carlo step (MCS), involves attempts to update $N$ randomly selected spins. The time evolution was followed, depending on the 
initial state, from $10^{3}$ up to $2 \times 10^{5} \mathrm{MCS}$. The magnetization, the autocorrelation, and the second moment of the magnetization were averaged over a number $n_{s}$ of samples with the same initial conditions but using different configurations. The $n_{s}$ values were in the range 80-6000, depending of the initial state and the segmentation step. In order to facilitate the lecture of the paper, $n_{s}$ is indicated in the figures.

In order to estimate the error of the evaluated parameters we have used a variant of the blocking method [24]. We fit the time dependence of each observable and check that the error that emerges from the fit verifies the following condition: if we repeat the fitting procedure for several independent sets of measurements the obtained values spread within the proposed error. We have found that the described procedure becomes stable if we disregarded an initial time that is specified in each case during the presentation of the results (we are implicitly assuming that the disregarded time interval includes the microscopic time defined above assuring STD validity).

\section{RESULTS AND DISCUSSION}

\section{A. Dynamic evolution from the ordered state}

Figure 2 shows the decay of the magnetization as a function of time obtained at different annealing temperatures for the segmentation step $k=5$. As can be observed, log-periodic oscillations are present in the time evolution of the magnetization over the full range of time and for all temperatures. We believe that the log-periodic structure exhibited by the data is a typical feature of systems with discrete scale invariance (DSI) [16]. So, in Sec. III C we first show that the topology of the substrate (the Sierpinki carpet in our case) possesses this symmetry property and subsequently, we formulate arguments linking the spatial characteristic length of the fractal to the time characteristic period of the dynamic behavior of the Ising system. Now, in order to account for our numerical observations we assume that the power-law behavior expected for the time evolution of the magnetization [Eq. (5)] should be replaced by

$$
M(t) \propto t^{-\beta / v z}\left[1+A \cos \left(\frac{2 \pi}{\log (P)} \log (t)+\phi\right)\right],
$$

where $\log (P)$ is a $\log$ arithmic period, while $A$ and $\phi$ are the amplitude and the phase of the oscillation, respectively. An expression, similar to Eq. (8) has been used by other authors in order to describe the time dependence of systems that

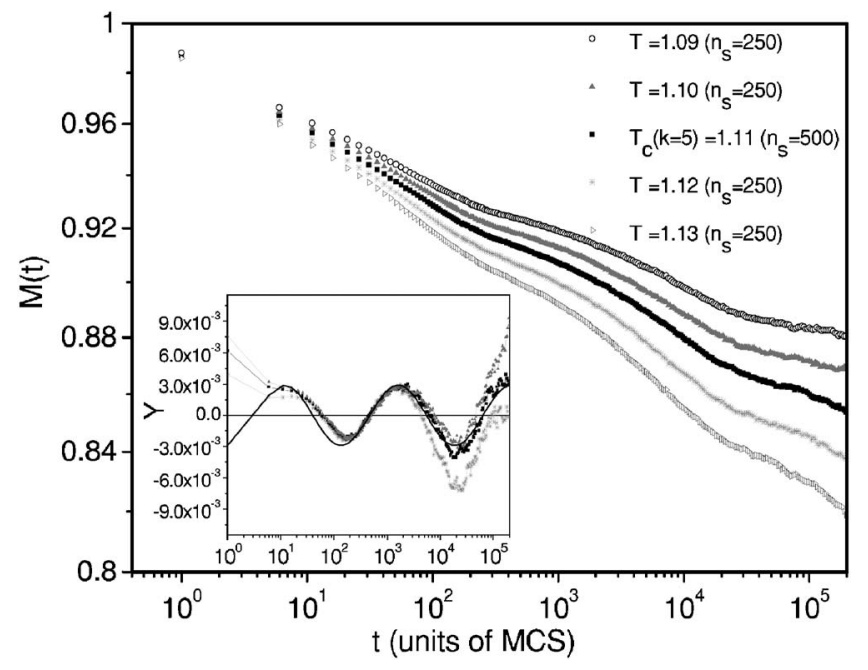

FIG. 2. Log-log plots of magnetization vs time obtained for different temperatures close to criticality, starting from ordered initial conditions $\left(m_{0}=1\right)$ for the segmentation step $k=5$. The effective critical temperature $T_{c}(k=5)=1.11(1)$ was determined by obtaining the best fit of $M(t)$ to Eq. (8). The inset shows the oscillating component of $M(t)\left(Y=\frac{M(t)-C t^{-\beta / v_{2}}}{C t^{-\beta / v_{z}}}\right)$ vs $t$ in linear-log scale for $T=1.11$, 1.10, and 1.12, which we fit with a cosine function (solid line). More details in the text.

exhibit a DSI in their dynamic behavior, such as the energy release on the approach of impending rupture, earthquakes [16], and biological systems (proteins) [19]. It should also be mentioned that the correction to the power law, introduced in Eq. (8), corresponds to the first term of the Fourier expansion of a logarithmic-periodic function.

In order to study the critical regime we settle down the temperature range that exhibits the smallest deviations from a power-law behavior, namely $1.09 \leqslant T_{c}(k=5) \leqslant 1.13$. Subsequently, we determine the critical temperature by finding the smallest deviation of the data from Eq. (8). This study yields $T_{c}(k=5)=1.11(1)$, where the error bar was assessed by considering the closest pair of temperatures that present noticeable but small deviations. The inset of Fig. 2 shows the oscillatory component of $M(t)$ that is nicely fitted by the cosine function for $T=1.11$, and also shows the departures from the cosine function for $T=1.10$ and 1.12. Logarithmic-periodic oscillations are also observed for the segmentation steps $k$ $=4$ and 6 , and the procedure previously described was also used to determine the corresponding effective critical temperatures, as reported in Table I (second column).

TABLE I. Effective critical temperatures and parameters obtained by fitting the time dependence of the magnetization according to Eq. (8): ratio of exponents $\beta / \nu z$, amplitude $(A)$, logarithmic period $[\log (P)]$, and phase $(\phi)$ of the oscillation. Data obtained by starting the simulations from an ordered initial state and for segmentation steps $k=4,5$, and 6 .

\begin{tabular}{cccccc}
\hline \hline$k$ & $\mathbf{T}_{c}(k)$ & $\beta / \nu z$ & $A$ & $\log (P)$ & $\phi$ \\
\hline 4 & $1.14(1)$ & $0.0145(1)$ & $0.0023(2)$ & $2.11(7)$ & $\pi$ \\
5 & $1.11(1)$ & $0.0116(1)$ & $0.0029(1)$ & $2.15(1)$ & $\pi$ \\
6 & $1.10(1)$ & $0.0110(1)$ & $0.0029(1)$ & $2.16(1)$ & $\pi$ \\
\hline \hline
\end{tabular}




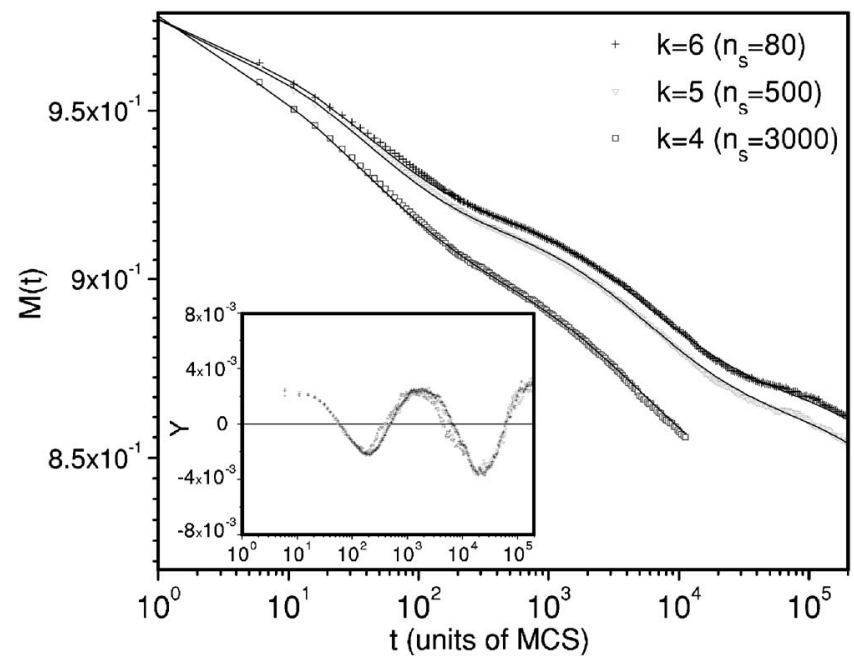

FIG. 3. Log-log plots of magnetization vs time obtained at the effective critical temperature starting from ordered initial conditions $\left(m_{0}=1\right)$ for segmentation steps $k=4,5$, and 6 . The corresponding fits are also shown by means of full lines. The inset shows the shape of the oscillation of $M(t)\left(Y=\frac{M(t)-C t^{-\beta / v z}}{C t^{-\beta / v z}}\right)$ vs $t$ in linear-log scale.

Figure 3 shows the magnetization decay at the effective critical temperature for the segmentation steps 4,5 , and 6 . The fits were carried out after disregarding an initial time interval $t_{\min }=30 \mathrm{MCS}$, and the obtained parameters are reported in Table I. For $k=4$, in order to assure that the nonequilibrium correlation length remains smaller than the system size, the time interval was from 30 to $10^{4} \mathrm{MCS}$. As observed in the inset of Fig. 3 the logarithmic-periodic oscillations of the magnetization decay have similar shape for $k$ $=5$ and 6 , but they are slightly different for $k=4$. The effective $T_{c}(k=6)=1.10(1)$ is in relatively good agreement with determinations performed by means of Monte Carlo simulations in equilibrium and obtained by applying finite-size scaling, namely $T_{c}<1.178$ [2] and $T_{c}=1.077(3)$ [25]. Furthermore, a careful inspection of the data reveals a systematic but convergent decrease in both $T_{c}(k)$ and $\beta / \nu z$ (Table I, second and third columns, respectively) when the segmentation step is increased, suggesting that our results could be taken as upper bounds. The exponent $\beta / \nu z$ for $k=6$ is notably smaller than both the accepted value for the Ising model in $d=2$, given by $\beta / \nu z=0.0577$ (3) [26], and our estimation for the $\operatorname{SC}(3,1)$, given by $\beta / \nu z=0.03412(7)$ [15]. In this way at the critical point a larger deviation from the translational symmetry, i.e., an increase of the lacunarity, causes the decay of the long-range order to slow down.

The logarithmic derivative of the magnetization with respect to $\tau$ is evaluated by taking the difference between the values of $M(t)$ at two temperatures close to the critical one $\left[T_{c}(k) \pm 0.01\right]$. The results of this calculation are shown in Fig. 4, where the logarithmic-periodic oscillations can be clearly observed for the segmentation step $k=6$. For $k=5$ the oscillations are also suggested by the behavior obtained for $t>10^{3} \mathrm{MCS}$. In order to obtain the exponent $1 / \nu z$, we propose the same correction for the power law as that applied to the magnetization and given in Eq. (8), namely

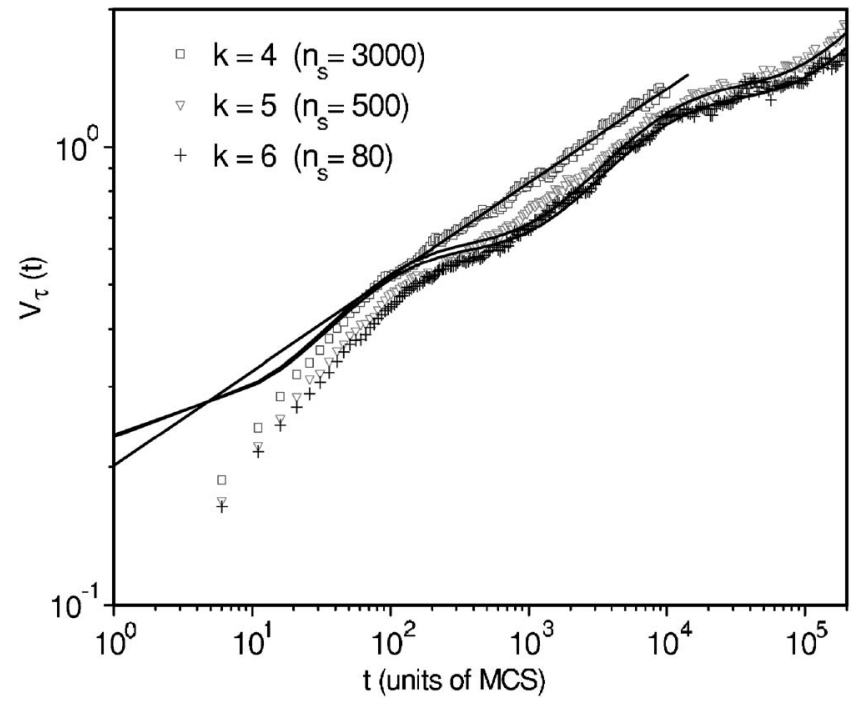

FIG. 4. Log-log plot of the logarithmic derivative of magnetization vs time obtained at $T_{c}(k)$ by starting from the ordered initial conditions $\left(m_{0}=1\right)$. Results obtained for three different segmentation steps as listed in the figure. The full lines correspond to fits obtained for $t>100 \mathrm{MCS}$, according to Eq. (9) for $k=5$ and 6, and to Eq. (6) for $k=4$, respectively. See details in the text.

$$
V_{\tau}(t) \propto t^{1 / \nu z}\left[1+B \cos \left(\frac{2 \pi}{\log (P)} \log (t)+\Phi\right)\right]
$$

where $B$ and $\Phi$ are the amplitude and the phase of the oscillations, respectively. The fits were performed within the range $10^{2}$ to $2 \times 10^{5} \mathrm{MCS}$ giving $\log (P)=2.0(2)$ for $k=5$ and 6 , while the results obtained for the exponents $1 / \nu z$ are listed in Table II. For $k=4$, the presence of oscillations cannot be detected within the time range considered, i.e., from $10^{2}$ to $10^{4}$ MCS. So, in order to give a crude estimation for the effective critical exponent, we fitted the data by using Eq. (6) (see Table II). Figure 5 shows the determination of the exponent $\frac{d_{\text {eff }}}{z}$ (see Table II) from the time dependence of the Binder cumulant, according to Eq. (7). Although a very weak oscillation is insinuated, the noise in the data hinders its analysis in a reliable way. The trend of the data for the exponents $1 / \nu z$ and $\frac{d_{e f f}}{z}$, namely a convergent decrease when $k$ is increased, is consistent with the previous observations re-

TABLE II. Effective critical exponents determined from the time dependence of the magnetization [second column, Eq. (8)], the Binder cumulant [third column, Eq. (7)], and the logarithmic derivative of magnetization [fourth column, Eq. (9)]. Also, the asterisk corresponds to a crude estimation performed by using Eq. (6). Data obtained by starting the simulations from an ordered initial state and for $k=4,5$, and 6 segmentation steps of the fractal.

\begin{tabular}{ccccc}
\hline \hline$k$ & $\beta / \nu z$ & $d_{\text {eff }} / z$ & $\frac{1}{\nu z}$ & $\beta$ \\
\hline 4 & $0.0145(1)$ & $0.556(3)$ & $0.209(2)^{*}$ & $0.069(1)$ \\
5 & $0.0116(1)$ & $0.54(1)$ & $0.167(8)$ & $0.069(3)$ \\
6 & $0.0110(1)$ & $0.54(3)$ & $0.162(8)$ & $0.068(3)$ \\
\hline \hline
\end{tabular}




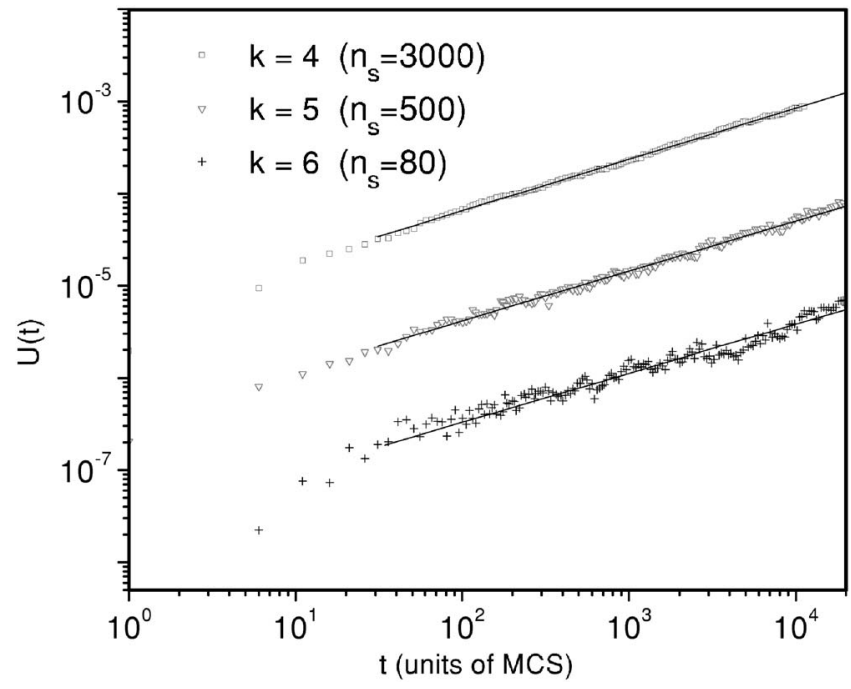

FIG. 5. Log-log plot of Binder's cumulant vs time obtained at criticality, starting from ordered initial conditions $\left(m_{0}=1\right)$, for $k$ $=4,5$, and 6 . The full lines correspond to the best fits obtained for $t>30 \mathrm{MCS}$.

ported for $\mathrm{SC}(3,1)[12,15]$ and strongly suggests that our results should be taken as reliable upper bounds.

From these results the order parameter critical exponent $\beta$ can be obtained, which appears to be less sensitive to the change of the segmentation step than other exponents shown in Table II. It is worth mentioning that our best estimation, given by $\beta=0.068(3)$, is significantly smaller than the values corresponding to the Ising model in $d=2[\beta=0.125$ (exact) and in $d_{H} \cong 1.8927$ on $\operatorname{SC}(3,1)[\beta=0.121(5)]$.

\section{B. Dynamic evolution from the disordered state}

The dynamic evolution after quenching the system to $T_{c}$ when the simulations are started from the disordered state (not shown) presented a weak dependence on temperature. This shortcoming hinders an independent estimation of $T_{c}(k)$ based on these measurements. However, by using the value of $T_{c}(k)$ obtained by means of simulations started from the ordered state, it is possible to obtain an independent evaluation of the critical exponents.

Figure 6 shows the initial increase in magnetization observed for the segmentation steps 4,5 , and 6 , obtained for different values of the small initial magnetization $\left(m_{0}=0.02\right.$, 0.04 , and 0.06). The data exhibit a weak dependence on the segmentation step as can be deduced from the overlapping of the curves. Within the time regime considered, the magnetization always increases and the data can be fitted to a power law, as expected from Eq. (2). Nevertheless, a soft curvature of the data can be observed for larger times due to the fact that $m_{0}$ is finite and the power law is actually expected to hold in the $m_{0} \rightarrow 0$ limit. So, in order to determine the exponent we performed a fit of the data within the time interval 30-100 MCS. As can be observed in the inset of Fig. 6, the exponents show a weak dependence on $m_{0}$. Then, the exponent $\theta$ was evaluated by a linear extrapolation to $m_{0}=0$. So, according to our results, listed in Table III, the exponent $\theta$ $=0.181(2)$ for the $\mathrm{SC}(4,2)$ fractal appears to be the same as

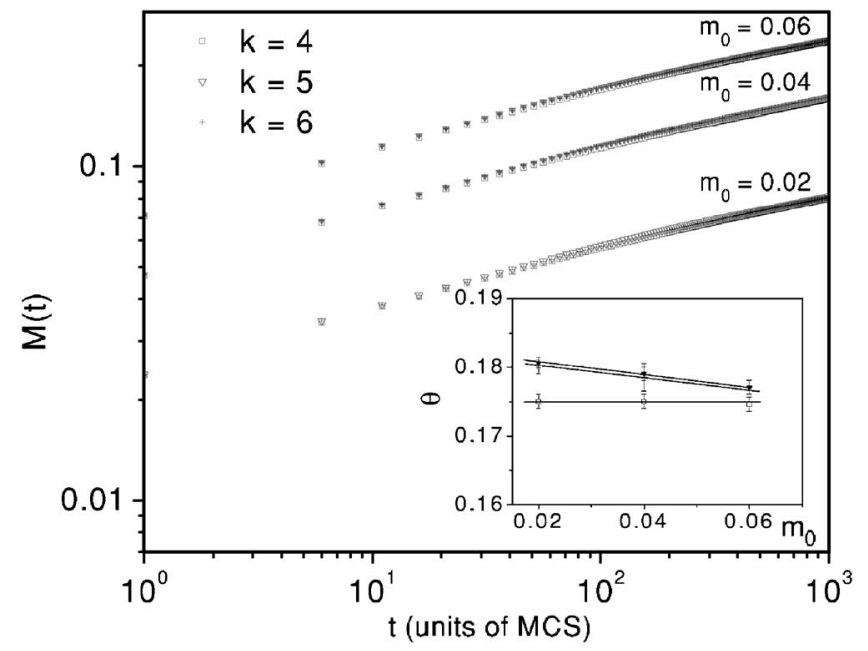

FIG. 6. Log-log plots of magnetization vs time obtained at the effective critical temperature, starting from disordered initial conditions slightly modified to obtain different values of the initial magnetization $m_{0}$. Data corresponding to $k=4\left(n_{s}=50000\right), 5\left(n_{s}\right.$ $=5000)$, and $6\left(n_{s}=500\right)$, and different values of $m_{0}$, which are also indicated in the figure. The inset shows the dependence of $\theta$ on the initial magnetization $m_{0}$ that allowed us to extrapolate the exponent $\theta\left(m_{0} \rightarrow 0\right)$ for each $k$.

that for $\operatorname{SC}(3,1)[\theta=0.1815(6)]$ and slightly smaller than the value for the Ising model in $d=2$, given by $\theta=0.191(3)$ [26].

Figure 7 shows the time evolution of the second moment of the magnetization, the interval was from 30 to $10^{4} \mathrm{MCS}$, according to Eq. (4). The obtained values are also listed in Table III, which are within the error bars independent of the segmentation step. The exponent $\gamma$ of the susceptibility can be estimated assuming that hyperscaling law $d_{e f f}=\frac{2 \beta}{\nu}+\frac{\gamma}{\nu}$ holds, and by combining the results corresponding to different initial conditions, i.e., using the exponents of the second moment and of the logarithmic derivative of the magnetization. The obtained values (see Table III) show a convergent increase with $k$, giving $\gamma=3.3(2)$ for $k=6$. This value is significantly larger than those obtained for the Ising model in $d=2$ and in SC $(3,1)$, namely $\gamma=1.75$ (exact) and $\gamma(k=6)$ $=2.22(2)$, respectively. However, it is considerably smaller than the values reported from the simulations performed in equilibrium and obtained by using finite size scaling, for the same segmentation step, which are $\gamma=5.39$ [25] and $\gamma$ $>5.73[10]$.

On the other hand, the decay of the autocorrelation function (see Fig. 8) slightly depends on the segmentation step. The fits were carried out after disregarding an initial time interval of $t_{\min }=30 \mathrm{MCS}$, since after that the power-law behavior expected from Eq. (4) is observed. The obtained exponents $\lambda=d_{e f f} / z-\theta$ are also reported in Table III. It is worth mentioning that by inserting the value of $\theta$ already determined from the initial increase in the magnetization (see Fig. 6 ) in the exponent of the autocorrelation function, one can also calculate $d_{e f f} / z$, as listed in the fourth column of Table III. The obtained results are in full agreement with independent determinations performed by fitting the time dependence of the Binder cumulant starting simulations from ordered states, see Table II. 
TABLE III. Exponents determined from the dynamic behavior of the second moment of the magnetization [second column, see Eq. (3)], the autocorrelation [third column, see Eq. (4)], and the initial increase in the magnetization [fourth column, see Eq. (2)]. Data obtained by starting the simulations from disordered initial states and for $k=4,5$, and 6 . From these exponents the values of $d_{e f f} / z, \beta / \nu z$, and $\gamma$ are estimated and are listed in columns 5 and 6 , respectively.

\begin{tabular}{ccccccc}
\hline \hline$k$ & $\left(d_{\text {eff }} / z\right)-(2 \beta / \nu z)$ & $\left(d_{\text {eff }} / z\right)-\theta$ & $\theta$ & $d_{\text {eff }} / z$ & $\beta / \nu z$ & $\gamma$ \\
\hline 4 & $0.532(5)$ & $0.389(8)$ & $0.175(2)$ & $0.564 .(8)$ & $0.016(9)$ & $2.55(3)$ \\
5 & $0.531(4)$ & $0.360(8)$ & $0.182(2)$ & $0.542(8)$ & $0.006(9)$ & $3.2(2)$ \\
6 & $0.529(5)$ & $0.360(9)$ & $0.181(2)$ & $0.541(9)$ & $0.006(10)$ & $3.3(2)$ \\
\hline \hline
\end{tabular}

Also, by inserting the exponent $d_{\text {eff }} / z$ in the expression of the exponent of the second moment of the magnetization, given by $d_{e f f} / z-2 \beta / \nu z$, one can obtain an additional estimation of $\beta / \nu z$, as listed in the fifth column of Table III. The values are smaller than those obtained for the magnetization decay corresponding to the evolution from the ordered state (see the second column of Table II). We attribute this difference to the small value of $\beta / \nu z$ relative to both $d_{\text {eff }} / z$ and $\left(\frac{d_{e f f}}{z}-\frac{2 \beta}{\nu}\right)$. In this way, a small relative error for these exponents induces a large error in $\beta / \nu z$, as shown in Table III.

\section{Discrete scale invariance in space and time}

Discrete scale invariance (DSI) has extensively been discussed by Sornette [16]. It is a weak kind of scale invariance according to which an observable $O(x)$, which is a function of a control parameter $x$, obeys the scaling law

$$
O(x)=\mu(b) O(b x),
$$

under the change $x \rightarrow b x$. Here $b$ is no longer an arbitrary real number as in the case of continuous scale invariance but it can only take specific discrete values, which in general have the form $b_{n}=\left(b_{1}\right)^{n}$, where $b_{1}$ is the fundamental scaling ratio.

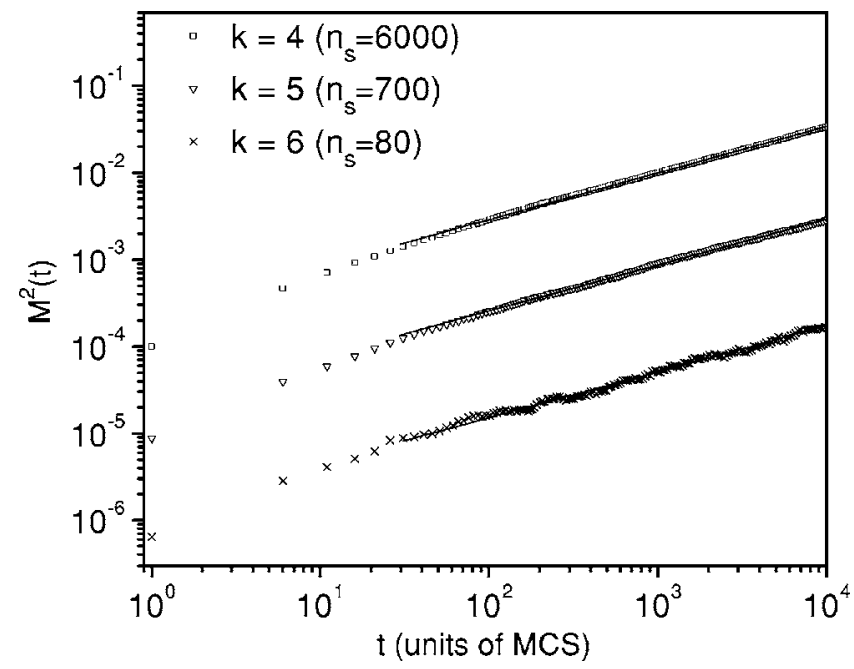

FIG. 7. Log-log plots of the second moment of magnetization vs time, obtained at the effective critical temperature, starting from disordered initial conditions with $m_{0}=0$. Data obtained by taking $k=4,5$, and 6 . The full lines indicate the fit of the data according to Eq. (3) for $t>30$.
It is easy to show that if an observable $O(x)$ satisfies Eq. (10) for an arbitrary $b$ it necessarily has to obey a power law of the type $O(x)=C x^{\alpha}$, where $\alpha$ is an exponent; but in the case of DSI the solution of Eq. (10) yields

$$
O(x)=x^{\alpha} F\left(\frac{\log (x)}{\log \left(b_{1}\right)}\right),
$$

where $F$ is a periodic function of period one.

To illustrate that the $\mathrm{SC}(4,2)$ leads up to DSI, let us calculate the dependence of the mass for this fractal on the distance $R$ to an arbitrary position $\mathbf{r}_{0}$ given by

$$
m\left(R, \mathbf{r}_{0}\right)=\int_{0}^{R} \int_{0}^{2 \pi} \rho\left(\mathbf{r}-\mathbf{r}_{0}\right) r d \theta d r ; \quad \rho(\mathbf{r})=\sum_{i=1}^{N} \delta\left(\mathbf{r}-\mathbf{r}_{i}\right),
$$

where $i$ runs over all the sites in the fractal and $\mathbf{r}_{\mathbf{i}}$ is the position of the $i$ th site. Note that, in principle, the mass depends on the point $\mathbf{r}_{\mathbf{0}}$ we are choosing as the coordinate origin. Figure 9 shows a log-log plot of the dependence of $m\left(R, \mathbf{r}_{\mathbf{0}}\right)$ on $R$, where $\mathbf{r}_{\mathbf{0}}$ is taken as one of the corners of the fractal. As can be seen in the inset, the mass oscillating component follows quite well the behavior expected from Eq. (11). The value for $b_{1}=4.01$ (2) obtained by the fit with four terms in the Fourier expansion coincides with the linear size of the generating cell, which of course is the fundamental scaling ratio. By choosing different positions $\mathbf{r}_{\mathbf{0}}$, the dependence of the mass on $R$ looks different than the one shown in Fig. 9. However, when we compute $m(R)$, the averaged mass function over all the sites $\mathbf{r}_{\mathbf{0}}$ in the lattice, we have found that it is still possible to factorize a periodic part $F\left[\log (R) / \log \left(b_{1}\right)\right]$ with $b_{1} \approx 4$. Therefore we conclude that the mass, an exclusively topological property of the fractal, presents a logarithmic-oscillatory behavior, this being a signature of the DSI of the fractal.

We would like to remark that the fitted value of the fundamental scaling ratio $b_{1}=4.01$ (2) for $k=5$ obtained from Fig. 9 is in excellent agreement with the exact value given by $b_{1}=4$. This result suggests a fast convergency of the logarithmic-periodic behavior towards the value corresponding to the mathematical fractal and gives us strong confidence in the values of the characteristic time evaluated by fitting the logarithmic-periodic dynamics for $k=6$ (see Table I). 


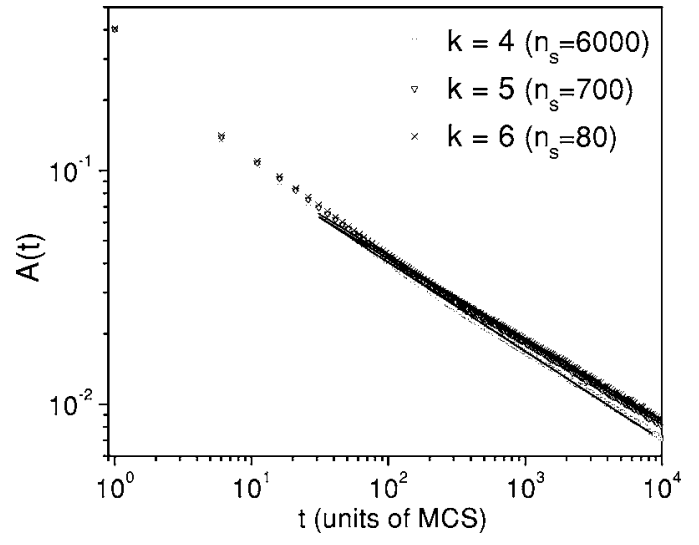

FIG. 8. Log-log plots of autocorrelation vs time, obtained at effective critical temperature, starting from disordered initial conditions with $m_{0}=0$ and by taking $k=4,5$, and 6 . The full lines indicate the fit of the data according to Eq. (4) for $t>30$.

The observed logarithmic-periodic oscillation in the decay of the magnetization (Figs. 2 and 3) leads us to abandon the standard power-law decay of the form $M(t) \propto t^{-\beta / \nu z}$ [Eq. (5)] and propose an ansatz based on Eq. (11), namely the typical power law modulated by a logarithmic-periodic function [see Eq. (8)]. Of course, by means of this assumption we implicitly recognize that the DSI intrinsic of the substrate is somewhat capable of influencing the dynamic behavior of the overlaying ferromagnet yielding to a kind of DSI in the time scale. Following this line of reasoning one has to admit that the fundamental scaling ratio between length scales $\left(b_{1}\right)$ of the fractal has to translate into an intrinsic ratio between time scales for the oscillations given by the period $P$. It is wellknown that for a critical system evolving towards equilibrium, characteristic spatial and temporal scales are linked through the dynamic exponent $z$, i.e., the development of the correlations up to a length $\xi$ scales as $\xi \propto t^{1 / z}$. Therefore going one step further in our speculation, we propose that the scaling ratios are also linked according to

$$
b_{1}=P^{1 / z} \text {. }
$$

In the Appendix we demonstrate under which assumptions this relation holds, but let us now show that Eq. (13) is consistent with our measurements. In fact, Eq. (13) implies $z=\frac{\log (P)}{\log \left(b_{1}\right)}$. Then, from Table I we take $\log (P)=2.16$ yielding $z \approx 3.6$. Also, the logarithmic derivative of the magnetization yields $\log (P)=2$, so one has $z \approx 3.3$. These rough estimations of the dynamic exponent can be compared to the accepted value for the 2D-Ising model, namely $z=2.165$ [26] and our previous estimation of $z=2.55$ [15] for the $\operatorname{SC}(3,1)$. So, all these results are consistent with an increasing trend of $z$ when the fractal dimension of the substrate decreases, anticipating the occurrence of a dramatic increase in the slowing down, characteristic of the second-order phase transitions, in low dimensionality. Furthermore, independent measurements (see Tables II and III) yield to $\frac{d_{\text {eff }}}{z} \approx 0.54$, so one has $d_{\text {eff }}$ $\approx 1.9(z \approx 3.6)$ and $d_{\text {eff }} \approx 1.8(z \approx 3.3)$, i.e., two figures in reasonable agreement with the fractal dimension of the $\operatorname{SC}(4,2)$ given by $d_{H} \cong 1.7925$. On the other hand, based on

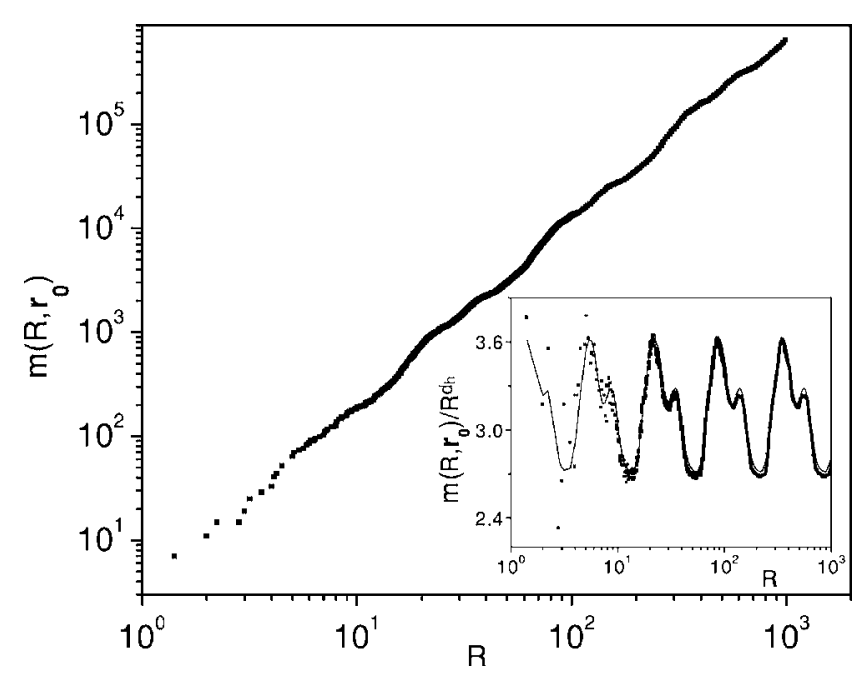

FIG. 9. Log-log plot of the mass $m\left(R, \mathbf{r}_{\mathbf{0}}\right)$ as a function of $R$, for the segmetation step $k=5$ of the $\operatorname{SC}(4,2)$. The origin $\mathbf{r}_{\mathbf{0}}$ has been chosen at one of the fractal corners. The inset shows the logarithmic-oscillatory part, $\left.F\left[\log (R) / \log \left(b_{1}\right)\right]=m\left(R, \mathbf{r}_{\mathbf{0}}\right) / R^{d_{H}}\right)$, and a fit with the first four terms of the Fourier expansion. For the fundamental scaling ratio we have obtained $b_{1}=4.01(2)$.

both the measured exponent $\frac{1}{\nu z}=0.162(8)$ and the crude estimation of $z$, we could give a very rough estimation of the correlation length exponent, which should be of the order of $\nu \approx 1.8$, also in agreement with the trend shown for the 2DIsing model $(\nu=1)$ and $\operatorname{SC}(3,1)(\nu \approx 1.39)$.

\section{CONCLUSIONS}

We have studied the nonequilibrium critical dynamics in the short-time regime of the Ising ferromagnet embedded in a fractal substrate, namely the $\mathrm{SC}(4,2)$. The influence of the fractal substrate on the dynamic evolution of some physical observables is clearly identified through at least two effects: (i) The dependence of the short-time regime on the segmentation step; and (ii) the occurrence of logarithmic-periodic oscillations superimposed to the power-law time behavior observed in both the decay of the magnetization and its logarithmic derivative with respect to the reduced temperature, when the system is annealed from $T=0$ to $T_{c}$. We propose and provide evidence to support that these oscillations are a consequence of the DSI of the fractal substrate.

In order to describe the decay of the magnetization upon annealing to $T_{c}$ we proposed an ansatz that involves the standard power-law behavior but now modulated by a logarithmic-periodic oscillatory function [see Eq. (8)]. This kind of function has also been used to describe the behavior of systems that exhibit a DSI in its dynamic behavior $[16,19]$. By fitting the data with Eq. (8) we determine $T_{c}(k$ $=6)=1.10(1)$, a figure that is in relatively good agreement with the values obtained by other authors by performing equilibrium measurements and by applying a finite-size scaling approach $[2,25]$.

The exponent $\theta=1.81(2)$ of the initial increase in the magnetization, determined for the segmentation step $k=6$, appears to be the same as that for the $\operatorname{SC}(3,1)$ 
$[\theta=0.1815(6)]$ but it is only slightly smaller than the accepted value for the Ising model in $d=2[\theta=0.191(3)]$, suggesting that this exponent is not significantly affected by the dimensionality of the substrate. It is worth mentioning that $\theta$ is related to $x_{0}$, such that $x_{0}=z \theta+\frac{\beta}{\nu}$ and that the former exponent sets the time scale for the initial increase in the magnetization through $t_{m i c} \simeq m_{0}^{-x_{0} / z}$.

The second moment of the magnetization and the autocorrelation function obtained by starting the dynamic evolution from the disordered initial state follow the expected powerlaw behavior. This fact allows us to perform an independent determination of the exponents $\frac{d_{\text {eff }}}{z}$ and $\frac{\beta}{\nu z}$, which turn out to be self-consistent with the values obtained from simulations starting from the ordered state.

We would like to remark that the effective critical exponents corresponding to all observables measured show a convergent trend when the segmentation step is increased, indicating that the largest segmentation used in this work $(k$ $=6$ ) would be considered a good approximation of the mathematical fractal.

Our estimation $\beta=0.068(3)$ indicates that the order parameter critical exponent for the $\mathrm{SC}(4,2)$ is smaller than that corresponding to the Ising model in $d=2(\beta=1 / 8)$, which is almost the same as that reported for the case as the $\operatorname{SC}(3,1)$ $[\beta=0.121(9)]$. On the other hand, our estimation for the exponent of the susceptibility $[\gamma=3.3(2)]$ is significantly larger than those corresponding to the 2D-Ising magnet $(\gamma=1.75)$ and the $\operatorname{SC}(3,1)$ for the same $k[\gamma=2.22(2)]$. Nevertheless, it is notably smaller than the values reported from equilibrium simulations using finite-size scaling $(\geqslant 5.39)[10,25]$.

We hope that the present work represents an extensive attempt to not only numerically characterize the relevant critical properties of the Ising model on a fractal substrate, but also to give clear evidence that the DSI of the underlying fractal structure influences dramatically the dynamic evolution of an Ising magnet by causing the occurrence of logarithmic-periodic oscillations. Of course, we have discussed the difficulties one encounters when dealing with these systems, but even more importantly our study allows us to clearly identify and characterize the influence of the fractal substrate on the power-law behavior expected for the nonequilibrium critical dynamics in the short-time regime.

Finally, we have shown that if DSI holds in space and time domains (as it emerges from the obtained results) and if the correlation length scales as $t^{1 / z}$, then the space and time scaling ratios of the corresponding DSI should be related by $P^{1 / z}=b_{1}$. This statement is further validated by independent calculations of dynamic exponent $z$ [27]. We think that the proposed interrelation between space and time symmetries goes beyond the studied Ising magnet on a fractal substrate and will be of importance also for the understanding of other nonequilibrium systems with discrete scaling symmetry.

\section{ACKNOWLEDGMENTS}

This work was supported by CONICET, UNLP, ANPCyT, and Fundación Antorchas (ARGENTINA). The A. von Humboldt Foundation (Germany) is greatly acknowledged for the provision of valuable computer equipment. Alberto Maltz and Ernesto Loscar are acknowledged for fruitful discussions.

\section{APPENDIX}

In this appendix we show that Eq. (13) can be straightly proven for a certain observable $M$, if one assumes the following.

(i) $M(t)$ obeys a time DSI of the form

$$
M(t)=\mu\left(P_{n}\right) M\left(P_{n} t\right), \quad P_{n}=P^{n},
$$

where $P$ is the period and $n$ is an integer.

(ii) The correlation length scales as

$$
\xi \propto t^{1 / z} \text {. }
$$

(iii) The same observable $M$, as a function of the correlation length, obeys a spatial DSI of the form

$$
\tilde{M}(\xi)=\widetilde{\mu}\left(b_{n}\right) \tilde{M}\left(b_{n} \xi\right), \quad b_{n}=b_{1}^{n},
$$

where $b_{1}$ is the fundamental scaling ratio between length scales and $\tilde{M}$ is used to denote $\tilde{M}(\xi)=M[t(\xi)]$.

Assumption (ii) implies $t=a \xi^{2}$ for some constant $a$. Then by replacing on the right-hand side of Eq. (A1) one obtains

$$
M(t)=\mu\left(P^{n}\right) M\left(P^{n} a \xi^{z}\right)=\mu\left(P^{n}\right) M\left[a\left(P^{n / z} \xi\right)^{z}\right],
$$

and writing $M(t)$ as $\tilde{M}(\xi)$ this equality becomes

$$
\tilde{M}(\xi)=\mu\left(P^{n}\right) \tilde{M}\left(P^{n / z} \xi\right)
$$

and the comparison with Eq. (A3) leads us to the following equalities:

$$
P^{n / z}=b_{1}^{n}, \quad \widetilde{\mu}\left(b_{1}^{n}\right)=\mu\left(P^{n}\right),
$$

and therefore $P^{1 / z}=b_{1}$.
[1] L. Hao and Z. R. Yang, J. Phys. A 19, 1627 (1987).

[2] P. Monceau and P. Hsiao, Physica A 331, 1 (2004).

[3] Y. Gefen, A. Aharony, and B. Mandelbrot, J. Phys. A 16, 1267 (1983).

[4] Y. Gefen, B. B. Mandelbrot, and A. Aharony, Phys. Rev. Lett. 45, 855 (1980).

[5] Y. Gefen, A. Aharony, and B. Mandelbrot, J. Phys. A 17, 1277
(1984).

[6] B. B. Mandelbrot, The Fractal Geometry of Nature (Freeman, San Francisco, 1982).

[7] J. F. Gouyet, Physics and Fractal Structures (Springer-Verlag, Paris, 1996).

[8] R. Blumenfeld and R. C. Ball, Phys. Rev. E 47, 2298 (1993).

[9] R. Blumenfeld and B. B. Mandelbrot, Phys. Rev. E 56, 112 
(1997)

[10] P. Monceau and M. Perreau, Phys. Rev. B 63, 184420 (2001).

[11] M. Perreau and J. C. S. Levy, Phys. Rev. A 40, 4690 (1989).

[12] G. Pruessner, D. Loison, and K. D. Schotte, Phys. Rev. B 64, 134414 (2001).

[13] H. K. Janssen, B. Schaub, and B. Schmittmann, Z. Phys. B: Condens. Matter 73, 539 (1989).

[14] B. Zheng, Int. J. Mod. Phys. B 12, 1419 (1998).

[15] M. A. Bab, G. Fabricius, and E. V. Albano, Phys. Rev. E 71, 036139 (2005).

[16] D. Sornette, Phys. Rep. 297, 239 (1998).

[17] J. C. Lessa and R. F. S. Andrade, Phys. Rev. E 62, 3083 (2000).

[18] M. F. Shlesinger and B. J. West, Phys. Rev. Lett. 67, 2106 (1991).

[19] T. Y. Shen, K. Tai, and J. A. Mc Cammon, Phys. Rev. E 63,
041902 (2001)

[20] H. Isliker and L. Vlahos, Phys. Rev. E 67, 026413 (2003).

[21] P. Monceau and P. Hsiao, Phys. Lett. A 300, 687 (2002).

[22] B. Zheng, M. Schulz, and S. Trimper, Phys. Rev. Lett. 82, 1891 (1999).

[23] I. Vattulainen, T. Ala-Nissila, and K. Kankaala, Phys. Rev. E 52, 3205 (1995).

[24] M. E. J. Newman and G. T. Barkema, Monte Carlo Methods in Statistical Physics (Clarendon Press, Oxford, 2001).

[25] J. M. Carmona, Umberto Marini Bettolo Marconi, J. J. RuizLorenzo, and A. Tarancón, Phys. Rev. B 58, 14387 (1998).

[26] B. Zheng, Physica A 283, 80 (2000).

[27] A precise calculation of the exponent $z$ would be very valuable in order to confirm quantitatively the validity of Eq. (13). Such a work is under progress for other Sierpinski carpets. 